\title{
Hypertrophic and Inflammatory Markers in Isoproterenol-Induced Cardiac Hypertrophy and its Pharmacological Correction
}

\section{Sukoyan GV ${ }^{*}$, Golovach SV1, Dolidze NM¹, Kezeli TD², Gongadze NV ${ }^{3}$, Chipashvili M² and Okujava MV ${ }^{3}$}

${ }^{1}$ International Scientific Centre of Introduction of New Biomedical Technology, Tbilisi, Georgia

2Javakhishvili State University, Pharmacological Department, Tbilisi, Georgia,

${ }^{3}$ Tbilisi State Medical University, Medicinal Pharmacological Department, Tbilisi Georgia

\begin{abstract}
Backgrounds: Short-term sustained beta-adrenergic stimulation is a hallmark of sympathetic hyperactivity and a common future in cardiovascular disease. Activation of endogenous cell signaling pathways that negatively regulate cardiac hypertrophy including the decline of NAD and the reduction of the intracellular NAD $/ N A D H$ ratio. The hypothesis that prevention of NAD depletion in myocardium may be critical in the treatment of cardiac hypertrophy and inflammatory responses was tested.

Methods: On the 7 days of isoproterenol (ISO)-induced cardiac hypertrophy $(\mathrm{CH})$ all animals were randomly assigned into 3 groups: control-without therapy (infusion of 0,9\% $\mathrm{NaCl}$ ); main I-receive $10 \mathrm{mg} / \mathrm{kg}$ nebivolol per os

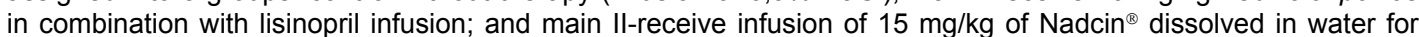
injection. All animals were euthanized throughout 7 days after beginning of treatment.

Results and conclusion: The increases of heart weight by $18,6 \%$ and heart-to-body weight ratio by $35,5 \%$ observed in ISO-induced $\mathrm{CH}$ were significantly suppressed in lisinopril-nebivolol therapy and monotherapy with $\mathrm{NAD}^{+}$containing drug, Nadcin ${ }^{\circledast}$. Therapy with Nadcin ${ }^{\circledast}$ restored the normal level of $\mathrm{NAD}^{+} / \mathrm{NADH}$ ratio and norepinephrine, in myocardium and cerebral arteries, decrease level of nuclear factor kappa B (NF-kB) and tumor necrosis factor (TNF)alpha in myocardium, cerebral arteries and blood and as a result blocking the myocardial hypertrophy progression. Treatment with $\mathrm{Nadcin}^{\circledR}$ on the ISO-induced model of cardiac injury in rabbits occurs significant anti-hypertrophic and anti-inflammatory effects and occurs preventive action on the redox-potential, NAD $/ \mathrm{NADH}$ decreasing and NAD depletion of myocardium and redox-potential decreasing in cerebral arteries. Thus, therapeutic strategy for NAD repletion have shown high therapeutic potential for cardiac, vascular and neurologic diseases.
\end{abstract}

Keywords: Cardiac hypertrophy; Cerebral arteries; Experiment modeling; NAD; Redox-potential; Tumor necrosis factor alpha; Nuclear factor kappa B

\section{Introduction}

One consequence of excessive catecholamine stimulation is an increased $\beta$-adrenergic receptor kinase activity leading to uncoupling of the decreased left ventricular myocardial $\beta$-adrenoceptor population in failing myocardium. Together with an increase in inhibitory G-proteins, this results in a decreased inotropic responsiveness of the heart to catecholamines. Chronic increased sympathetic activation represents an important hallmark of induced maladaptive (pathologic) hypertrophy development, resulting in myocyte cell death, fibrosis, ventricular dilation, as independent cause of its transition to heart failure [1-4]. In cardiac hypertrophy $(\mathrm{CH})$ and $\mathrm{CHF}$, the G-dependent pathways activated by norepinephrine, as one of neurohormones, stimulate mitogen-activated protein kinases (MAPK); the elusive signaling pathway responsible for many cerebral and brain injury. Despite of that the common link between $\mathrm{CH}$, endothelial dysfunction, MAPK activation and cerebrovascular events still speculative [5,6]. Several experimental and clinical data suggest that left ventricular (LV) hypertrophy is associated with cerebral damage even in the absence of clinical symptoms $[2,3,7]$. These hypertrophic stimuli affect the not only a mere response to the mechanical stress from elevated blood pressure, but also dysbalance in some factors (signaling small molecules) promoting the progression of LV pathological remodeling and vasoactive peptides releasing (such as norepinephrine, angiotensin II and endothelin-1), growth factors, oxidative stress markers, heat shock proteins, calcineurin, cytokines and some kinases. Although this diverse array of $\beta$-adrenergic stimulated interacting cascades was shown to induce cardiac remodeling, it is not determined yet whether chronic $\beta$-adrenergic stimulation alters genes or proteins expression in remote vascular bed, such as the cerebral arteries, with possible contribution in development of cerebrovascular events $[2,7,8]$. The intracellular $\mathrm{NAD}^{+} / \mathrm{NADH}$ ratio controls flux through various dehydrogenase enzymes involved in both anaerobic and aerobic metabolism and also regulates posttranslational protein modification. The intracellular $\mathrm{NADP}^{+} / \mathrm{NADPH}$ ratio controls flux through the pentose phosphate and the polyol pathways, while also regulating ion channel function and oxidative stress progression $[9,10]$. Not only does the $\mathrm{NAD}^{+} / \mathrm{NADH}$ ratio regulate the rates of $\mathrm{ATP}$ production, it can also modify energy substrate preference and depletion of cellular NAD levels and deterioration in NAD-mediated activation of Silent Information regulator (SIRT)3, but not SIRT1 cascade mechanism $[9,11,12]$. The exogenous NAD restored the activity of endogenous anti-hypertrophic signaling via activation of the SIRT3-liver kinase B (LKB) 1-AMP kinase (AMPK) pathway [6]. Experiments carried out to demonstrate the mechanism of cardioprotective effects of NAD

${ }^{*}$ Corresponding author: Galina V Sukoyan, International Scientific Centre of Introduction of New Biomedical Technology, 19, Kairskaya str, 0137, Tbilisi Georgia, Tel: +995322319344/+995595418030; E-mail: galinasukoian@mail.ru

Received September 08, 2017; Accepted October 16, 2017; Published October 23, 2017

Citation: Sukoyan GV, Golovach SV, Dolidze NM, Kezeli TD, Gongadze NV, et al (2017) Hypertrophic and Inflammatory Markers in Isoproterenol-Induced Cardiac Hypertrophy and its Pharmacological Correction. Cardiovasc Pharm Open Access 6: 225. doi: 10.4172/2329-6607.1000225

Copyright: () 2017 Sukoyan GV, et al. This is an open-access article distributed under the terms of the Creative Commons Attribution License, which permits unrestricted use, distribution, and reproduction in any medium, provided the original author and source are credited. 
showed that it prevented the hypertrophic response by shutting down pro-hypertrophic Aktl (proteinkinase B) signaling, and maintaining the activity of the anti-hypertrophic AMPK signaling pathway. Repeated or continuous injection of the $\beta A R$ agonist isoproterenol (ISO) in animals induces marked $\mathrm{CH}$ within 7 days without systolic hypertension [13], making it a useful tool in experimental model. ISO interacts with $\beta$ ARs of cardiomyocyte sarcolemmal membrane, leading to the activation of different intracellular signaling pathways. $\beta$-Adrenergic stimulation leads to activation of many signals that modulate the expression of proto-oncogenes, such as c-fos, c-jun, and c-myc, with subsequent induction of $\mathrm{CH}[7,13,14]$. Previously, we provided evidence that prolonged ISO infusion in a rabbit model leads to functional impairment of the coronary arteries, which implies a novel mechanism for reduced coronary reserve during $\mathrm{CH}[3,15]$. Since full understanding of this signaling pathway is the first step for better intervention of $\beta \mathrm{AR}$-induced $\mathrm{CH}$ and reliable interpretation of possible involvement in cerebrovascular events. To the best of our knowledge, this reports represents the first application of oxidized form NAD-containing drug, Nadcin ${ }^{\circledR}$, as an inhibitor of cardiac hypertrophic signaling via the prevention of myocardium NAD depletion mechanism and its remote impact on the cerebral arteries. The contribution of acute innate immune response nuclear factor $\mathrm{kB}$ (NF-kB) and tumor necrosis factor (TNF)- $\alpha$ and in this cascade in myocardium and cerebral arteries were also tested.

\section{Materials and Methods}

\section{Experimental design}

Practically healthy chinchilla rabbits weighting $2.3 \pm 0.5 \mathrm{~kg}$ maintained on the standard fed ad libitium, normal-protein diet and had free access to water were used in the study. All animals were handled in compliance with the European Convention on Animal Care and received humane care in accordance with the Principles of Laboratory Animal Care and the Guide for the Care and Use of Laboratory Animals (published in the Official Daily N. L358/1-358/6, 18 December 1986), and the US National Institutes of Health (Guide for the Care and Use of Laboratory Animals, NIH publication no. 85-23). Animals were housed individually in a humidity- and temperature-controlled room with a 12-hour light cycle light/dark cycle at temperature of $22^{\circ} \mathrm{C}$. The experiments conducted according to protocols approved by the local committee on the use of laboratory animals of the International Research Centre of Introduction of New Biomedical Technology. After a 2-week acclimatization period they were randomly divided into two groups, one designated to control $(\mathrm{n}=10)$ and the other to become ISOinduced $\mathrm{CH}(\mathrm{n}=40)$. Cardiac hypertrophy was induced by daily ISO (Sigma Chemicals Co.) administration $(10 \mathrm{mg} / \mathrm{kg}$ body weight, i.v.) for 7 days [8]. The control group was treated similarly with $0.9 \%$ saline (2 $\mathrm{ml} / \mathrm{kg}$ body weight). The mortality in ISO-treated group was $16 \%$ and zero in control group. Twenty seven rabbits with $\mathrm{CH}$ were randomly assigned into follows groups relative to receive therapy: $\mathrm{CH}$, control II-without therapy (receive physiological solution, $\mathrm{n}=10$ ); main I-daily given orally lisinopril (inhibitor of angiotensin converting enzyme, in form of lisinopril-dihydrate, Sigma-Aldrich), $1,0 \mathrm{mg} / \mathrm{kg}$ body weight, in combination with nebivolol hydrochloride (a cardioselective beta 1-adrenoceptor blocker facilitating vascular release of nitric oxide (NO), $10 \mathrm{mg} / \mathrm{kg}$ per day given orally via gastric lavage for 10 days starting on day 7-th after chronic ISO-induced myocardial damage $(n=12)$; and main II-receive $15 \mathrm{mg} / \mathrm{kg}$ body weight, of $\mathrm{Nadcin}^{\circledR}$, first oxidized form NAD-containing drug with antiischemic and anti-inflammatory activities [16-18], manufacturing by "Biotechpharm GE", Georgia] $(\mathrm{n}=11)$. Treatment duration in both group was 10 days. The number of survivors in each group was recorded daily until the end of study. Surviving rabbis were observed daily, and their weights were recorded weekly. After the defined treatment period, the rabbits were euthanized (by an i.p. injection of $50 \mathrm{mg} \cdot \mathrm{kg}^{-1}$ pentobarbitone sodium) by approval of Ethical Committee for Animal Care (Department ) protocol, samples of heart and total cerebral arteries (e.g., anterior, middle and posterior) were quickly removed, and crushed under liquid nitrogen and used for tissue analyses. Blood samples will be collected and serum and plasma will be separated and stored at $-70^{\circ} \mathrm{C}$ and thawed just before use.

\section{Assessment of cardiac hypertrophy}

The heart weight index was calculated by dividing the heart weight by the body weight in each group and as a cardiac wet-to-dry weight ratios. Wet-to-dry weight ratios were determined by drying tissue at $70^{\circ} \mathrm{C}$.

\section{Biochemical parameters study}

The NAD and NADH levels were measured in frozen hearts and cerebral arteries after the extracted by two freeze/thaw cycles or homogenization in $400 \mu \mathrm{l}$ of $\mathrm{NAD}^{+} / \mathrm{NADH}$ Extraction Buffer (BioVision, Mountain View, CA, USA). The homogenate was filtered using $10 \mathrm{kDa}$ cut-off filters. The NAD content was calculated from the standard curve and normalized to the protein content of the sample. The Bradford method (Coomassie brilliant blue G-250 staining) was used to detect protein content and calculate the protein excretion at $24 \mathrm{~h}$. Norepinephrine (NE) concentration in plasma and tissue was assayed using fluorometric methods and measured at $400 \mathrm{~nm}$ excitation and $510 \mathrm{~nm}$ emission wave-lengths. Serum TNF- $\alpha, N F-k B$ were determined by ELISA technique using standard kits (RayBiotech, Inc., USA) and brain natriuretic peptide (BNP) using MyBioSource ELISA assay.

\section{Statistical analysis}

The statistical significance assessed as the mean \pm standard deviation (SD) using Student t test for normally distributed variables and $\mathrm{p}<0.05$ considered significant. Ch-squire testing used the analyze categorical data. Correlation between estimated cardiac hypertrophy parameters and redox-potential and inflammatory markers were investigated by Spearman's correlation test. All statistical calculations were performed using the Statistical Sciences (SPSS, version 9, Russia).

\section{Results}

\section{Cardiac deremodeling in short-term induced heart hypertrophy and its pharmacological correction}

There were no statistically significant differences of body weight gain in either group. The increases in heart weight (HW) by $18,6 \%$ and heart-to-body weight ratio by $35,5 \%$ observed for the ISO group were suppressed by $6,4 \%$ and $9,7 \%$ respectively after lisinopril-nebivolol therapy and by 15,8 and $31,5 \%$ in $\mathrm{Nadcin}^{\circledR}$-treated group. 7 days duration ISO-induced hypertrophy resulted in increased of both right (control: $1,51 \pm 0,10$ vs ISO-treated rabbits: $1,84 \pm 0,11 \mathrm{mg}, \mathrm{p}<0.05$ ) and left ventricles ( $\mathrm{LV}$, control: $2,84 \pm 0,11$ vs ISO-treated rabbits: $3,44 \pm 0,10 \mathrm{mg}, \mathrm{P}<0.01$ ). The ratios of $\mathrm{LV}$ weight to $\mathrm{HW}$ increased by $20,5 \%$, left ventricular thickness by $18,2 \%$ and interventricular septum thickness increase by $12,4 \%$ in ISO-induced $\mathrm{CH}$, and cardiac wet-to-dry weight ratio by $6,7 \%$, in comparison with healthy control group (Table 1). Thus, 7-days ISO-induced is accompanied with small extent of oedema and a small increased in marker of heart failure development, BNP, up to $18 \mathrm{pmol} / \mathrm{ml}(24,4 \mathrm{pmol} / \mathrm{ml}$ in heart failure with decreased of pump-function model of rabbits heart failure). 


\begin{tabular}{|c|c|c|c|c|}
\hline \multirow[b]{2}{*}{ Parameters } & \multirow[b]{2}{*}{$\begin{array}{l}\text { Control I, (practically healthy } \\
\text { animals), } n=10\end{array}$} & \multicolumn{3}{|c|}{ Chronic ISO-induced myocardial damage } \\
\hline & & $\begin{array}{l}\text { Control II, } 0.9 \% \mathrm{NaCl} \\
n=10\end{array}$ & $\begin{array}{c}\text { Main I+lisinopril +nebivolol, } \\
\mathrm{n}=12\end{array}$ & $\begin{array}{c}\text { Main II+ Nadcin } \\
\mathrm{n}=11\end{array}$ \\
\hline Body weight. kg & $2.45 \pm 0.10$ & $2.21 \pm 0.12$ & $2.22 \pm 0.37$ & $2.52 \pm 0.16^{\#}$ \\
\hline Heart weight. g & $5.39 \pm 0.10$ & $6.39 \pm 0.12^{* *}$ & $5.98 \pm 0.14^{* \#}$ & $5.38 \pm 0.10^{\#++}$ \\
\hline Left ventricle weight. $g$ & $2.84 \pm 0.11$ & $3.44 \pm 0.18^{* *}$ & $3.15 \pm 0.09^{*}$ & $2.97 \pm 0.09^{\# \#}$ \\
\hline Right ventricle weight. g & $1.51 \pm 0.10$ & $1.84 \pm 0.11^{*}$ & $1.75 \pm 0.09^{*}$ & $1.57 \pm 0.06^{\#++}$ \\
\hline Heart: body ratio $\times 10^{-3}$ & $2.20 \pm 0.18$ & $2.98 \pm 0.12^{* *}$ & $2.69 \pm 0.09^{* \#}$ & $2.13 \pm 0.09^{\#+}$ \\
\hline LV: heart ratio & $0.39 \pm 0.03$ & $0.47 \pm 0.02^{*}$ & $0.45 \pm 0.08$ & $0.40 \pm 0.03^{\#}$ \\
\hline Cardiac wet-to-dry weight ratio & $5.41 \pm 0.16$ & $5.77 \pm 0.12^{\star}$ & $5.57 \pm 0.11^{\star}$ & $5.42 \pm 0.11^{\#}$ \\
\hline Relative LV mass. $\mathrm{mg} / 100 \mathrm{~g}$ body weight & $0.22 \pm 0.06$ & $0.36 \pm 0.06^{\star}$ & $0.33 \pm 0.05^{\star}$ & $0.24 \pm 0.05^{\star \#}$ \\
\hline Ventricular wall thickness. $\mathrm{mm}$ & $4.12 \pm 0.16$ & $4.87 \pm 0.15^{* *}$ & $4.70 \pm 0.16^{*}$ & $3.97 \pm 0.18^{\#+}$ \\
\hline Interventricular septum thickness. $\mathrm{mm}$ & $3.78 \pm 0.09$ & $4.25 \pm 0.16^{*}$ & $4.15 \pm 0.10^{*}$ & $3.88 \pm 0.12^{* \#}$ \\
\hline BNP. pmol/ml & $4.5 \pm 2.1$ & $18 \pm 3^{* * *}$ & $12.4 \pm 2.4^{\star * * \#}$ & $6.1 \pm 2.4^{\#+++}$ \\
\hline
\end{tabular}

Note: ${ }^{ \pm}$standard deviation, symbols; - significance of difference between the group; "with control, " with the ISO-induced cardiac hypertrophy; ${ }^{+}$with the nebivolol-lisinopril treated groups; one symbol-p<0.05, two-p $<0.01$; three- $p<0.001$.

Table 1: Changes in hypertrophic parameters in short-term ISO-induced cardiac hypertrophy and its pharmacological correction.

The LV-to-HW ratio and relative LV mass (because there is only the tendency to decrease the cardiac-wet-to dry weight ratio), ventricular wall thickness and interventricular septum thickness did not decreased after 7-days treatment with lisinoprol-nebivolol combination. The both characteristics of LV hypertrophy, cardiac-wet-to-dry weight ratio and relative LV mass returns to the normal level in Nadcin -treated animals. 7 -days course of Nadcin occurs positive effects on the ventricular wall and interventricular septum thickness and fully normalized the LV and RV weight and heart and LV-to body ratio. These simultaneously with reversed the increasing BNP content in blood confirmed the conclusion that Nadcin reverse the $\mathrm{CH}$ in condition of ISO-induced short-term hypertrophic damage of myocardium in rabbits. In this stage of $\mathrm{CH}$ myocardium is characterized the diminished of NE level in myocardium and its rise about two fold in blood and in less extent (in 1,2 time) in cerebral arteries. Elevation of NE level in plasma associated with middle increased in BNP indicated that ISO-induced myocardial damage was caused with moderate form of heart failure. Compared with the control group, rabbits in the main group treated with Nadcin more than after treatment with lisinopril and nevibolol were improved color and hair, restored the feeding, behavior and movement, decreased the cyanosis in the mouth and nose, fatigue, mild edema in the feet and paws.

Decreasing of redox-potential and elevation of level of norepinephrine and markers of inflammation in myocardium, cerebral arteries and blood under short-term ISO-induced cardiac hypertrophy in rabbits

ISO-induced 7-days duration $\mathrm{CH}$ is associated with the deterioration in redox-potential NAD/NADH in myocardium by $34 \%$ and in cerebral arteries by $36 \%$ besides the more pronounced decrease in pool of NAD in myocardium. Decreasing of redox-potential accompanied with the deterioration in the immune system response: hyperproduction of proinflammatory cytokines and markers of functioning of innate immune system NF-kB under ISO-induced hypertrophy. The NF-kB activity increased by $158 \%$ in myocardium and by $150 \%$ in cerebral arteries; the level of TNF- $\alpha$ increased and exceeds normal level in 11fold in plasma, in 10.7-fold in myocardium and in 2.5-fold in cerebral arteries (Table 2). More beneficial action of Nadcin in the prevention of cardiac maladaptive hypertrophy formation associated with the increased of NAD level in myocardial tissue by $25 \%$ and NAD/NADH ratio was raised by $32 \%$ and reach normal level in myocardium. In cerebral arteries in ISO-induced $\mathrm{CH} 7$ days duration content of NAD deceased by $15 \%$, pool of NADH increased by 14 and as a result redoxpotential decreased by $25 \%$. Treatment with Nadcin that leads to restoration of level of oxidized form NAD and redox-potential restores levels of NE and TNF- $\alpha$ in plasma, myocardium and cerebral arteries, and the activity of NF-kB in myocardial and cerebral arteries tissue. There were no correlation between the level of NE and TNF- $\alpha$ in plasma $(\mathrm{r}=0,37, \mathrm{NS})$, myocardium ( $\mathrm{r}=0,37, \mathrm{NS})$, and cerebral arteries $(\mathrm{r}=0,41$, $\mathrm{NS}$ ), or between NE and level of NF-kB (p65) activity in myocardium $(\mathrm{r}=0,51, \mathrm{NS})$ and cerebral arteries $(\mathrm{r}=0,50, \mathrm{NS})$. The strongly negative correlation between decreased of NAD/NADH level and increased the activity of NF-kB and the level of TNF- $\alpha$ in myocardium $(r=-0,72$, $\mathrm{p}<0,001$ and $\mathrm{r}=-0,77, \mathrm{p}<0,001)$, and cerebral arteries $(\mathrm{r}=-0,79, \mathrm{p}<0,001$, $\mathrm{r}=-0,81, \mathrm{p}<0,001)$, and NAD/NADH and TNF- $\alpha$ in plasma $(r=0,72$, $\mathrm{p}<0,001$ ), have been shown. Close relationship between changes in pool of NAD and production TNF- $\alpha$ confirmed the hypothesis that level of redox-potential controlled and regulated post-translantional step in TNF- $\alpha$ synthesis [12]. Obtained results delineate a novel relationship between metabolism and inflammatory response and a novel strategy for reversed the dysbalance in neuro-immune axis in ISO-induced damage of myocardium. These mediators partly help to perpetuate ventricular dysfunction, thus having an important role in prognosis. The treatment with $\beta$-adrenoblockers and ACE-inhibitors occurs neither significantly affected the activity of NF-kB and content of TNF- $\alpha$ in plasma, myocardium and cerebral arteries on day 7 of therapy, besides the decreased of level of NE in plasma and its increased in myocardium.

\section{Discussion}

Cardiac damage is physiological and pathological states of the heart which involves deterioration of complex molecular processes that lead to cardiomyocyte growth involve membrane receptors, second messengers, and transcription factors. At the molecular level cardiac hypertrophy is a consequence of imbalance between the activities of pro- and anti-hypertrophic molecules. Even though this could be a compensatory response initially to normalize increased wall tension of the ventricles, sustained increase in hypertrophy leads to ventricular dilatation and heart failure. The possibility of stopping or reversing pathologic cardiac hypertrophy and, thereby, slowing the development of heart failure is a topic of considerable clinical interest. Current therapy of cardiac hypertrophy progression are mainly based on the reduction of heart rate (beta-adrenoblockers) and cardiac overload and remodeling (ACE, mineralocorticoid antagonists, vasodilators), 


\begin{tabular}{|c|c|c|c|c|}
\hline \multirow{2}{*}{ Parameters } & \multirow{2}{*}{$\begin{array}{c}\text { Control I, } \\
n=10\end{array}$} & \multicolumn{3}{|c|}{ Chronic ISO-induced myocardial damage } \\
\hline & & Control II, n=10 & Main I, n=12 & Main II, n=11 \\
\hline \multicolumn{5}{|c|}{ Blood, plasma } \\
\hline $\mathrm{NAD}, \mathrm{nMol} / \mathrm{ml}$ & $19.7 \pm 0.8$ & $17.3 \pm 0.6^{*}$ & $17.1 \pm 0.7^{\star}$ & $20.0 \pm 0.6^{\#+}$ \\
\hline $\mathrm{NADH}, \mathrm{nMol} / \mathrm{ml}$ & $19.0 \pm 0.8$ & $23.0 \pm 1.0^{*}$ & $22.2 \pm 0.8^{*}$ & $19.9 \pm 0.6^{\star \#}$ \\
\hline NAD/NADH ratio & $1.04 \pm 0.06$ & $0.75 \pm 0.06$ & $0.77 \pm 0.05$ & $1.00 \pm 0.06$ \\
\hline Norepinephrine, $\mathrm{ng} / \mathrm{ml}$ & $1.10 \pm 0.08$ & $2.12 \pm 0.10^{* *}$ & $1.53 \pm 0.09^{* \#}$ & $0.92 \pm 0.08^{\# \#++}$ \\
\hline TNF-a, pg/mg & $1.2 \pm 0.2$ & $13.4 \pm 1.3^{* *+*}$ & $9.9 \pm 1.2^{*+*}$ & $2.5 \pm 0.4^{\# \#+++}$ \\
\hline \multicolumn{5}{|c|}{ Myocardium, LV } \\
\hline NAD, nMol/mg protein & $6.8 \pm 0.6$ & $5.1 \pm 0.6$ & $5.4 \pm 0.4$ & $6.5 \pm 0.5^{\# \#++}$ \\
\hline $\mathrm{NADH}, \mathrm{nMol} / \mathrm{mg}$ protein & $6.5 \pm 1.0$ & $7.4 \pm 0.8$ & $7.1 \pm 0.5$ & $6.9 \pm 0.4^{\#+1++}$ \\
\hline NAD/NADH ratio & $1.05 \pm 0.09$ & $0.69 \pm 0.06$ & $0.76 \pm 0.06$ & $0.94 \pm 0.05^{\# \#+++}$ \\
\hline Norepinephrine, $\mathrm{mg} / \mathrm{g}$ wet weight heart & $0.62 \pm 0.08$ & $0.31 \pm 0.06$ & $0,92 \pm 0.08$ & $0.70 \pm 0.05^{\# \#+++}$ \\
\hline TNF-a, pg/mg & $1.23 \pm 0.08$ & $13.0 \pm 0.3$ & $10.1 \pm 0.1$ & $2.12 \pm 0.18^{\# \#++}$ \\
\hline NF-kB(p65) activity, o.e. at $450 \mathrm{~nm}$ & $0.12 \pm 0.04$ & $0.31 \pm 0.06$ & $0.30 \pm 0.07^{* *}$ & $0.14 \pm 0.04^{\ldots++}$ \\
\hline \multicolumn{5}{|c|}{ Cerebral arteries } \\
\hline $\mathrm{NAD}, \mathrm{nMol} / \mathrm{mg}$ & $7.5 \pm 0.6$ & $6.3 \pm 0.4^{*}$ & $6.4 \pm 0.4^{*}$ & $7.1 \pm 0.4^{\#+}$ \\
\hline $\mathrm{NADH}, \mathrm{nMol} / \mathrm{mg}$ & $7.3 \pm 1.0$ & $8.4 \pm 0.6$ & $8.2 \pm 0.4$ & $7.5 \pm 0.5$ \\
\hline NAD/NADH ratio & $1.01 \pm 0.08$ & $0.75 \pm 0.06$ & $0.78 \pm 0.06$ & $0.95 \pm 0.06^{\# \#++}$ \\
\hline Norepinephrine, $\mu \mathrm{g} / \mathrm{g}$ wet tissue & $2.14 \pm 0.10$ & $2.56 \pm 0.10^{*}$ & $2.45 \pm 0.08^{*}$ & $2.09 \pm 0.07^{\# \#++}$ \\
\hline TNF-a, pg/mg & $2.9 \pm 0.2$ & $5.4 \pm 0.9^{*+*}$ & $4.9 \pm 0.7^{\star \star}$ & $2.6 \pm 0.3^{\ldots+\ldots+++}$ \\
\hline NF-kB(p65) activity, o.e. at $450 \mathrm{~nm}$ & $0.10 \pm 0.02$ & $0.25 \pm 0.05^{* *}$ & $0.22 \pm 0.03^{*}$ & $0.13 \pm 0.02^{\# \#++++}$ \\
\hline
\end{tabular}

Note: ${ }^{ \pm}$Standard deviation; -significance of difference between the group; "with control; "with the ISO-induced cardiac hypertrophy; ${ }^{+}$with the nebivolol-lisinopril treated groups; one symbol-p<0.05; two-p $<0.01$; three-p<0.001.

Table 2: Changes in the level ofredox-state, norepinephrine and markers of inflammation in short-term ISO-induced cardiac hypertrophy and its pharmacological correction.

which contribute to spare energy consumption, but do not address the issue of bioenergetic failure directly. New therapeutic tools to activation of endogenous cell signaling pathways that reverse a global alteration in bioenergetics systems are of particular interest as for the management of cardiac hypertrophy and heart failure. Pathologic hypertrophy occurs and hypoxia-ischemia progression not only in myocardium, but in cerebral arteries and resulted in increased NAD turnover due to raised activity of NAD-consuming enzymes such as poly(ADP-ribose) polymerase-1 and/or decreased activity of NAD salvage pathways, with a net result of depletion of intracellular NAD levels. NAD and/or NAD/NADH is essential for maintaining proper levels of NE in the synapses of neurons: after Norepinephrine crosses the neuron's synapses it loses one electron to form oxidized NE. In the presence of NAD, this oxidized NE then re-converts back to active NE. If there is a deficiency of NAD, the oxidized NE loses another electron to irreversibly form Noradrenochrome. Rapid glycolysis during neuronal activation increases the conversion of pyruvate to lactate and an increased $\mathrm{NADH} / \mathrm{NAD}^{+}$ratio, which in turn increases cerebral blood flow [8]. Loss of NAD can make a cell unable to carry out its energy-dependent functions and defend itself against oxidative stress because of loss of activity of certain cell-survival factors that are NAD-dependent, such as sirtuins [9]. Treatment with exogenous NAD blocked the induction of atrial natriuretic factor release from nuclei, thus suggesting the antihypertrophic activity of NAD [6]. The results indicated that phenylephrine (PE, $20 \mu \mathrm{M})$-mediated activation of these promoters was significantly reduced by treatment of cells with NAD, thus again demonstrating the anti-hypertrophic potential of NAD $[6,19,20]$. Early, Sukoyan has shown that treatment with oxidized form of NAD (preparation of Nadcin ${ }^{\circledR}$ ) reversed ischemic-reperfusion injury of myocardium in experimental models [16-18] and Bokeria confirm these results in randomized controlled study in patients with unstable angina pectoris and bypass grafting [21]. After this Bruzzone [22], have provided evidence that exogenous NAD enters into cardiomyocytes via connexin $43(\mathrm{Cx} 43)$ channels permeable to extracellular NAD, NAD treatment dramatically decrease astrocytes and neurocytes death induced by a genotoxic agents or ischemic and traumatic damage, markedly decrease autophagy in the brain transient ischemia in experiments. This study in vivo model of ISO-induced cardiac hypertrophy we showed that pathologic $\mathrm{CH}$ is associated with depletion of cellular NAD levels. Nadcin ${ }^{\circledast}$, stable medicine for the first time containing oxidized form $\mathrm{NAD}$, course treatment restored the cellular NAD levels and blocked the beta-adrenergic agonistmediated $\mathrm{CH}$ response. Nadcin abolished the increase in poly(ADP-ribose)-polymerase activity in the ischemic area of the right ventricle, nonischemic region, and ischemic area of the LV (by 2.4, 2.9 , and 1.52 times, respectively) and normalized bioenergetic activity of cardiomyocytes during ischemia-reperfusion myocardial injury [16]. These data also indicated that hypertrophy of cardiomyocytes may result from loss of cellular NAD levels and decreased redoxpotential, NAD/NADH and Nadcin ${ }^{\circledR}$ should be able to rescue cells from developing hypertrophy. To test this hypothesis, we treated the 7-days duration ISO-induced $\mathrm{CH}$ without pronounced congestion with Nadcin in comparison with traditional combination of ACE-inhibitors and $\beta$-adrenergic blockers. The results showed that ISO-induced $\mathrm{CH}$ caused depletion of cellular (myocardial and cerebral arteries in less extent, but significant) NAD levels by nearly $25 \%$. Addition of exogenous NAD in form of $\mathrm{Nadcin}^{\circledR}$ was capable of rescuing cells from this NAD loss. Sustained adrenergic stimulation is an important hallmark of the maladaptive hypertrophy that leads to elevated level of NF-kB and TNF- $\alpha$ in the heart and confirming the close link between inflammation and cardiac hypertrophy. Upregulation of NF-kB (p65) in cerebral arteries by $130 \%$ and TNF- $\alpha$ by $86 \%$ were observed also. On the other hand, treatment with Nadcin, unlike combination of nebivolol-lisinopril, exhibited significant decrease in TNF- $\alpha$ level in blood and downregulaion of cardiac and cerebral arteries tissue $\mathrm{NF}-\mathrm{kB}$, and close linear negative correlation between decreasing in NAD/NADH and increasing activity of NF-kB (p65) of cardiac $(\mathrm{r}=0,71, \mathrm{p}<0,001)$ and cerebral arteries $(\mathrm{r}=0,68, \mathrm{p}<0,01)$ has been 
Citation: Sukoyan GV, Golovach SV, Dolidze NM, Kezeli TD, Gongadze NV, et al. (2017) Hypertrophic and Inflammatory Markers in IsoproterenolInduced Cardiac Hypertrophy and its Pharmacological Correction. Cardiovasc Pharm Open Access 6: 225. doi: 10.4172/2329-6607.1000225

Page 5 of 5

observed. These results suggest that Nadcin attenuating ISO-induced inflammation. Recent studies provide evidence that increased in the level of NAD and redox-potential NAD/NADH via the inhibition of Nod-like receptors, including NLRP3, activation directly throughout regulation of $\mathrm{p} 65$ subunit of NF-kB by regulation the deacetylating cycle of SIRT1. A fall in NAD level and is more correctly the decrease the redox-potential NAD/NADH in myocardium, resulting in decreased the activity of SIRT1, will therefore promote the activation of NF-kB [23]. According to the results on patients with ischemic heart disease, arterial hypertension (II-III severity degree), rhythm and different genesis conduction disturbance, included of Nadcin into conventional mode of treatment leads to decreasing of angina functional class by $23 \%$, rarity of angina attack cases by $78 \%$, reduction of depth of STsegment and T-wave on the ECG and improvement of endothelial function. Cyanosis disappears in $95 \%$ of cases and myocardial demand for oxygen decreases by 30\% [21]. Good tolerance to Nadcin noted. This is accompanied (unlike the group which was not given Nadcin') by elevation of oxidation-reduction potential of pyridine nucleotide system and their absolute number, normalization of NADH-oxidase and decrease of endothelin-1 up to its normal level. Nadcin helps to restore thrombocyte membrane resistance to oxidation, reduce the level of malone dyaldehyde, increase the activity of catalase and superoxide dismutase $[19,21]$. The rejuvenation of the concept of NAD treatment came from studies of Araki who showed that exogenous addition of NAD to neurons delayed axonal degeneration in response to mechanical or chemical damage.

\section{Conclusion}

One novel approach of the rational strategy of cessation of $\mathrm{CH}$ and associated with this cerebral vessels injury progression is the activation of endogenous cell signaling pathways that negatively regulate $\mathrm{CH}$. Such first pharmacological exogenous agents that can facilitate the activity of these pathways is $\mathrm{Nadcin}^{\circledR}$, which is a modern medicine from the new therapeutic tools of replenishment for the management of $\mathrm{CH}$ and heart failure. At the cellular level various signaling mechanisms have been described that lead to development of $\mathrm{CH}$. Although the research on the effect of exogenous NAD in preventing diseases remains in its infancy, the significance of maintaining intracellular levels of NAD for cell protection gained momentum with the discovery of NAD-dependent deacetylases. Our data supports the therapeutic value of exogenous NAD, and show for the first time that it also has the potential to block $\mathrm{CH}$ response.

\section{Acknowledgment}

We thank the Chief Kamkamudze R. and his staff for animal housing. We express appreciation for E.Tsivtsivadze, PhD, Director of "Biotechpharm GE", Ltd Georgia, for the provision of preparation Nadcin ${ }^{\circledR}$ for investigation.

\section{References}

1. Coote JH, Chauhan RA (2016) The sympathetic innervation of the heart: Important new insights. Autonomic Neuroscience: Basic and Clinical 199: 17-23.

2. Selvetella G, Notte A, Maffei A (2003) Left ventricular hypertrophy is associated with asymptomatic cerebral damage in hypertensive patients. Stroke 34: 1766-1770.
3. Shin E, Soo KK, Rhee BD (2014) Different effects of prolong $\beta$-adrenergic stimulation on heart and cerebral artery. Integrative Medicine Research 3: 204-210.

4. Bisping E, Wakula P, Poteser M, Heinzel FR (2014) targeting cardiac hypertrophy: toward a causal heart failure therapy. J Cardiovasc Pharmacol 64: 293-305.

5. Kishi T (2014) Heart failure as an autonomic nervous system dysfunction. J Cardiol 59: 117-122.

6. Pillai VB, Sundaresan NR, Gupta MP (2014) Regulation of Akt Signaling by Sirtuins. Its implication in cardiac hypertrophy and aging. Circ Res 114: 368-378.

7. Kim HK, Park WS, Warda M (2012) Beta-adrenergic overstimulation impaired vascular contractility via actin-cytoskeleton disorganization in rabbit cerebral artery. PLoS One 7: e43884.

8. Gordon GR, Choi HB, Rungta RL (2008) Brain metabolism dictates the polarity of astrocyte control over arterioles. Nature 456: 745-749.

9. Abdellatif M (2012) Sirtuins and pyridine nucleotides. Circ Res 111: 642-656.

10. Ying W, Wei G, Wang D (2007) Intranasal administration with NAD profoundly decreases brain injury in a rat model of transient focal ischemia. Frontiers in Bioscience 12: 2728-2734.

11. Araki T, Sasaki Y, Milbrandt J (2004) Increased nuclear NAD biosynthesis and SIRT1 activation prevent axonal degeneration. Science 305: 1010-1013.

12. Van GF, Galli M, Gueydan C (2009) Intracellular NAD levels regulated TNF-a protein synthesis in a sirtuin-dependent manner. Nat Med 15: 206-210.

13. Tanaki M (2012) Cardiac mechanoenergetics for understanding isoproterenolinduced ratheart failure. Physiology 19: 163-170

14. Kim N, Chung J, Kim E, Han J (2003) Changes in the $\mathrm{Ca}^{2+}$-activated $\mathrm{K}^{+}$channels of the coronary artery during left ventricular hypertrophy. Circ Res 93: 541-547.

15. Sawyer DB, Siwik DA, Xiao L (2002) Role of oxidative stress in myocardial hypertrophy and failure. J Mol Cell Cardiol 34: 379-388.

16. Sukoyan GV, Kavadze IK (2008) Effect of Nadcin on energy supply system and apoptosis in ischemia-reperfusion injury to the myocardium. Bull Exp Biol Med 146: 321-324

17. Sukoyan GV, Andriadze NA, Guchua El, Karsanov NV (2005) Effect of NAD on recovery of adenine nucleotide pool, phosphorylation potential, and stimulation of apoptosis during late period of reperfusion damage to myocardium. Bull Exp Biol Med 139: 46-49.

18. Gongadze N, Rogava M, Bochorishvili T, Popova A (2012) Pharmacocorrection of redox-potential as a new therapeutic targets for the treatment of patients with chronic stable angina pectoris and diabetic mellitus. European Congress of Pharmacology PP: 71-73.

19. Gongadze N, Rogava M, Kezeli T (2013) Cardio-hepatic syndrome and therapeutic efficacy of NAD-containing drug. Clinical Therapeutics 35: e105-106.

20. Pillai VB, Sundaresan NR, Kim G (2010) Exogenous NAD blocks cardiac hypertrophic response via activation of the SIRT3-LKB1-AMPK pathway. J Biol Chem 285: 3133-3144.

21. Bockeria LA, Malikov VE, Arzumanyan MA (2008) Pharmacological correction of endothelial dysfunction and disturbance of structured organization of thrombocyte membranes in ischemic heart disease. Clinical Physiol Circul 1: 39-44.

22. Bruzzone S, Guida L, Zocchi E (2001) Connexin 43 hemi channels mediate Ca2+-regulated transmembrane NAD+ fluxes in intact cells. FASEB 15: 10-12.

23. Yeung F, Hoberg JI, Ramsey CS (2004) Modulation of NF-kB dependent transcription and cell survival by the SIRT1 deacetylase. EMBO 23: 23692380. 